Full Proceeding Paper

\title{
INTERNET OF VEHICLES-BASED REAL-TIME VEHICLE TRACKING
}

\author{
RAGHUNATH REDDY YEDDULA ${ }^{1}$, VETRIVELAN P2 \\ ${ }^{1}$ Department of Computing Science and Engineering, VIT University, Chennai, Tamil Nadu, India. ${ }^{2}$ Department of Computing \\ Science and Engineering, School of Electronics Engineering (SENSE), VIT University, Chennai, Tamil Nadu, India. Email: \\ asha.s@vit.ac.in \\ Received: 23 January 2017 , Revised and Accepted: 33 March 2017
}

\begin{abstract}
The leading-edge vehicle tracking and scanning system based on embedded board is intended and executed to observe any vehicle from different locations. Every year, many numbers of vehicles are stolen. To prohibit thievery, a phenomenal type of negator is preowned, like padlock, disk break lock and more which is a defensive motion, but it is not safe for all designs. The main impartial is to initiate a control entity that shows the locale spot of a vehicle proving by global position system (GPS) to find the position. Here, global system for mobile (GSM) is accepting for communicating with the vehicle that easily finding a steal. The entity is built for trying out the mastery of the locale spot that transmits to the user when the motion is in exciting direction and stable in the city and some other areas. This whole entity is built by bringing together a Raspberry Pi with GPS, general packet radio service (GPRS), and GSM. The preferred entity makes better use of unique automation that based on the Raspberry Pi. This entity works on the GPS and GSM SIM 900A modules which consists all the three things for communication. GPS and GPRS circulate the chasing information to the server to transmit the message to the mobile number which is saved in this sector for communicating through GSM. This preferred entity also acquires the traveler's protection by testing liquefied petroleum gas effused gas sensor MQ135 and temperature DS18B20 sensor.
\end{abstract}

Keywords: Raspberry Pi, Sensors, Global position system, global system for mobile modules.

(C) 2017 The Authors. Published by Innovare Academic Sciences Pvt Ltd. This is an open access article under the CC BY license (http://creativecommons. org/licenses/by/4. 0/) DOI: http://dx.doi.org/10.22159/ajpcr.2017.v10s1.19559

\section{INTRODUCTION}

Vehicle tracking entity's vital aim was to provide safety to the vehicles. Accident avoid system's aim is to rescue people in accidents. This entity improves safety for the vehicles. The recent modules such as global position system (GPS) are highly useful now-a-days, this system permits the owner to notice and trace the vehicle and finds the vehicle action and its motion.

This accident alert entity catches the accident and finds the location of the accident occurred area and sends GPS coordinate paths to the particular owners mobile, etc. Fire detector circuit used to reveal fire in the vehicle if the temperature reaches above the certain limit. Among all the new leading techniques GPS is the most used machinery and which gives very accurate report of locale spot in every condition and at every time. In the area of mobile communications, global system for mobile (GSM) is the most advanced mechanics. $85 \%$ of the global mobile market is using the GSM standard. In more than 220 countries, all over 2.5 billion people uses GSM. Therefore, every subscribed customer is able to utilize mobile communication worldwide by aligning international roaming network providers.

\section{LITERATURE SURVEY}

The use of this proposed entity is simple that children or illiterate persons can also use that. When a person wants to transfer the private message to another person, then they only have to type the text on key board. It is very secure because only an authorized person can access the actual message and the other user who want to hack or interprets the signal, receives only fake message and for decryption they want the exact codes used for the encryption. Only an authorized person knows about the actual decryption codes and able to debug it. Hence, it is secure and also an easy method for message transmission.

GPS-based vehicle tracking system is implemented by fetching the information of the vehicle such as distance and location using GSM and GPS. The information of the vehicle is obtained after every interval of time states by the provider. Then, this information transmitted to scanning or tracking server.

In addition to that the whole entity can be obtained using fieldprogrammable gate array (FPGA) also. A sensor named as accelerometer is used to catch any type of accidents and also triggers much kind of signals in case of any wrong activity. In this, FPGA spartan processor is used which manages all parts of the system according to the program written.

\section{PROPOSED SYSTEM}

\section{Objectives}

- Implements the GPS-based tracking and navigation entity

- Designing vehicle direction finder by proving the data report through GSM entity with the sequential steps:

- Taking require data from the motion direction at the specified interval of time

- Transfer of direction instruction data to track or scan the server

- Interfacing the display panel by utilizing Google earth to shows vehicle direction in the maps.

Block diagram (Fig. 1)

\section{Description}

In this proposed work setup, an embedded system is preowned to locate the vehicle motion through GPS and GSM modules.

Fig. 1 shows Raspberry Pi 3 connected with sensors and modules are used for combining different types of hardware components. The current design is an embedded setup, which allows continuous monitoring of vehicle and gives the data of the vehicle on a particular request.

Raspberry Pi 3 is connected through serial communication to the GSM and GPS receivers. GSM module is used to transfer the location in latitude and longitude paths of the motion at a certain area. The GPS 
module continuously sends the report, i.e., the longitude and latitude by intimating the various paths of the vehicle.

This module acquires various instructions as the output, but only the information getting out is captured and displayed onto the liquid crystal display. The same data are transmitted to the mobile at the other side from where the location of the motion is found.

Furthermore, the designed entity dispenses student's protection through the MQ 135 liquefied petroleum gas (LPG) (Fig. 2) gas effusion and DS18B20 temperature sensors (Fig. 3). These sensors combines through Raspberry $\mathrm{Pi} 3$. If the internal temperature of the vehicle reaches a particular threshold level or if any smoke gets effused inside the vehicle, the warning text will be transmit to the vehicle's owner. Like this, alert path will be provided by the system.

The whole setup can be interfaced through the Raspberry Pi 3 (Fig. 4) by connecting it with the various modules and sensors for decreasing the accidents and thefts occurring in many parts of the world. Accelerometer is used to avoid the accidents by giving some signals in case of any wrong movement. This design is much simple and less cost than others and we can stop our stolen vehicle using smart android application from our standing position in any part of the area. If there is any fuel leakage in the vehicle, it can be controlled using the gas sensor and sending message to the particular person.

This design can be operated in various logins for various persons such as:

- Owner's login

- Parent's login

- Student's login.

These various logins can be operated using android smart application through Google earths. This setup is used for finding the latitude and longitudes of the vehicle direction by fixing the particular sensors in the vehicles. Infrared sensors also used for

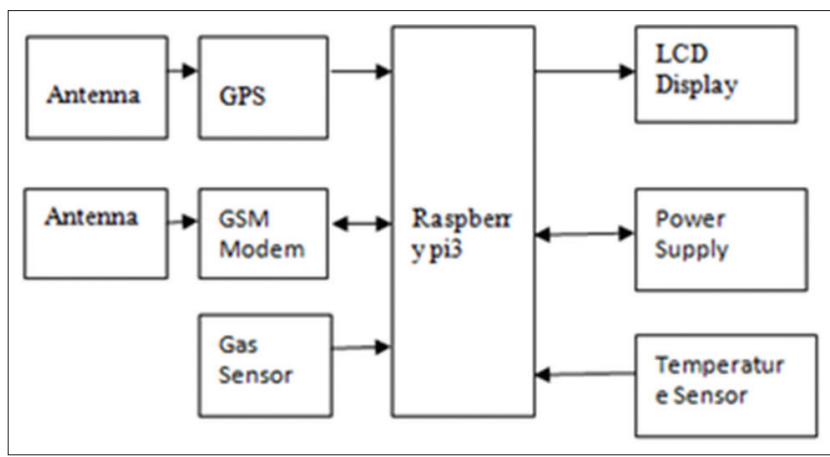

Fig. 1: Block diagram of the proposed system

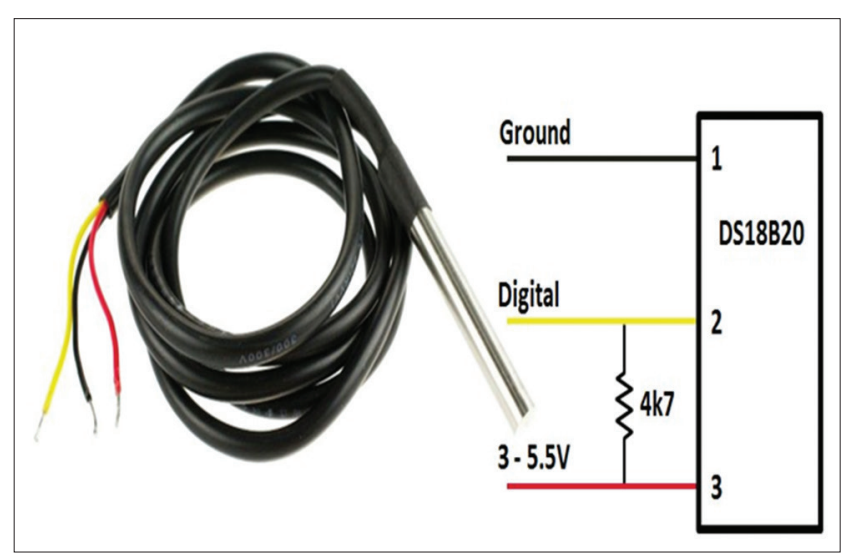

Fig. 2: DS18B20 temperature sensor this design for detecting the vehicles to avoid accidents and wrong paths of the vehicles.

\section{System specification}

1. Embedded board:

Features of Raspberry Pi 3 board:

- 40 pin extended GPIO

- $5 \mathrm{~V}$ maximum power supply through adaptor

- 1 GB RAM

- $4 \times$ USB 2 ports

- $\quad$ BCM43143 Wi-Fi on board

- Broadcom BCM2837 64 bit ARMv7 Quad Core Processor mechanized by single board system which amble the $1.2 \mathrm{GHz}$

- Full size high-definition multimedia interface

- Micro SD port slot for loading the system and for storing the data

- CSI camera port for interfacing with Raspberry Pi camera

- 4 pole stereo outputs and composite video ports

- Bluetooth low energy on board.

2. Sensors

i. Temperature sensor DS18B20

Features of temperature sensor DS18B20:

- Thermometer resolution is very selectable

- Allows -55 to $+125^{\circ} \mathrm{C}$ temperature

- User describable nonvolatile alarm backdrop

- There is no requirement of independent peripherals

- It can be mechanized from info line; power supply range varies from 3.0 to $5.5 \mathrm{~V}$

- $\quad$ Every device come across with own unique 64 bit serial code to store in the board ROM

- Unique 1 wire intermix depends on the one port pin for communication.

ii. MQ135 gas sensor

Features of MQ 135 LPG gas sensor

- $\quad$ Sensing resistance is $30-200 \mathrm{k}$

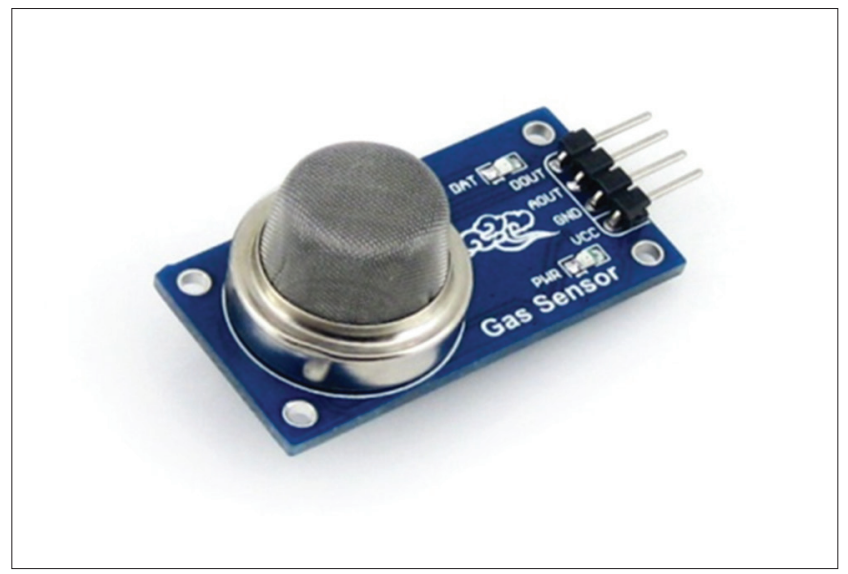

Fig. 3: MQ135 liquefied petroleum gas sensor

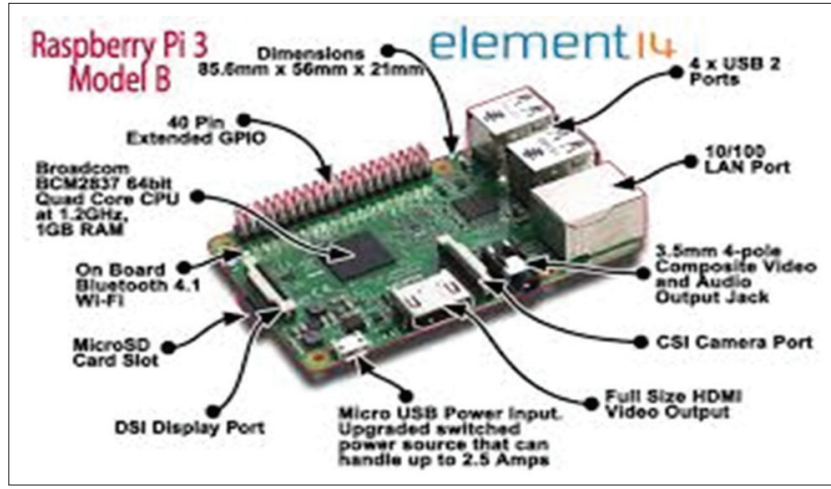

Fig. 4: Raspberry Pi 3 board 
- $\quad$ Concentration slope rate is $\leq 0.65$

- The given output is in dual mode

- Analog output with expanding concentration (the higher the concentration level, the higher the voltage)

- Branches of steam from sulfide and benzene, smoke, and other gases with more sensitivity

- It has reliable and long-life stability.

3. GSM module SIM900A

Features of SIM900A GSM module

- Dual-band operating speed is $900 / 1800 \mathrm{MHz}$

- TTL data with TX, RX, ground pins

- Empower with speaker and mic sockets

- Configurable baud rate

- GSM antenna with SMA adaptor

- $\quad$ Power controlled using 29302 WUIC

- Ultra-speed sensitivity - $165 \mathrm{dBm}$.

- Assisted GPS support for fast time to first fix

- 12 multitone active interposition canceller

\section{System design}

This system can be designed by interfacing Raspberry Pi 3 with GPS/ GSM/general packet radio service modules (Fig. 5) and LPG gas sensor MQ135 (Fig. 3) is connected to board through VCC and ground pins to the embedded board. DS18B20 temperature sensor (Fig. 2) is interfaced with board through $+5 \mathrm{~V}$, data, ground pins connecting to the Raspberry Pi board through $5 \mathrm{k}$ resistor. This vehicle tracking design takes data through GPS and transmits data through the GSM module (Fig. 5) to the particular mobile or laptop applying for mobile communication. Vehicle tracking entity is one of the most useful methodological advancements to scan the motion of the vehicle. The immunity system uses GPS, to capture the location of the tracing or captured vehicle and then avail

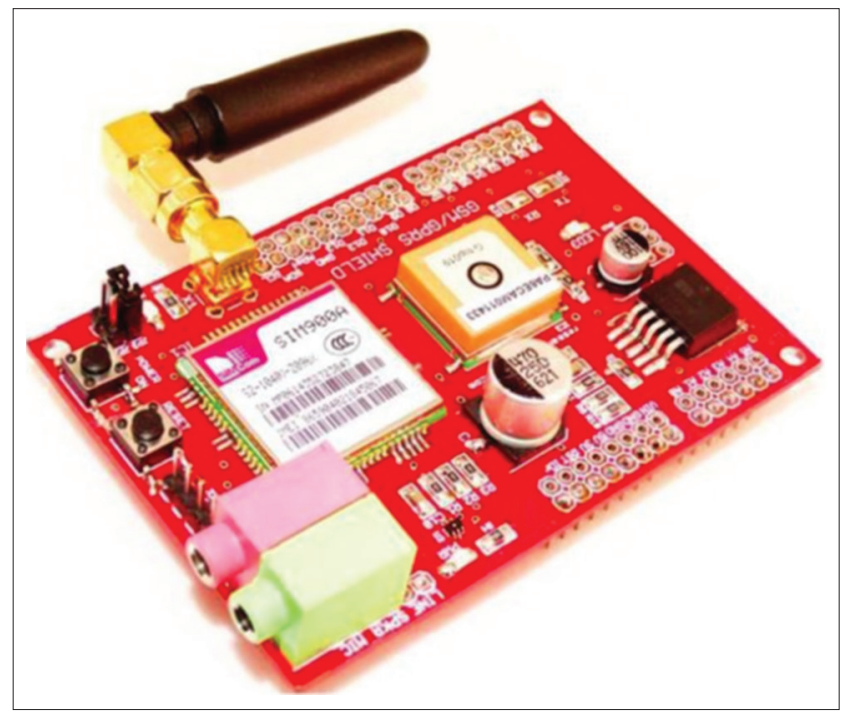

Fig. 5: Global system for mobile module SIM900A

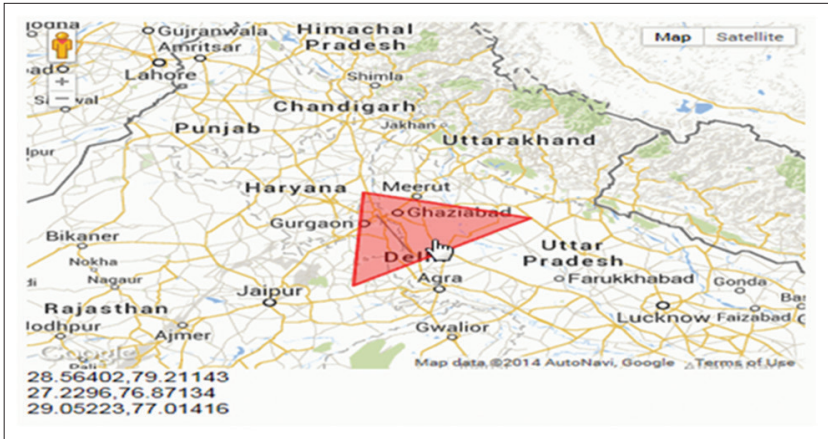

Fig. 6: Latitude and longitudes path using Google maps satellite to transfer the coordinate paths and the captured data to the display system. At the displaying entity, different softwares are used to find the vehicle on a Google maps. By these ways, the vehicle owners are alert to trace their own vehicles on a real-time environment formula. Due to this real-time tracking efficiency, vehicle tracking entities are becoming rapidly more preferred by the owners of costly wheels.

Vehicle owners can operates this total setup using android application that can be interfaced with the entity fixed in the vehicle and able to stop the vehicle from the particular position. From the prevention of using this application by others, the primary and guest logins can be created. All these data can be accessed through the Google maps by latitude and longitudinal paths of the particular area of the vehicle. Using Google maps (Fig. 6), the tracking location of the vehicle can be found.

Fig. 6 illustrates the latitude and longitudinal paths of the vehicle where it is moving using Google maps.

\section{IMPLEMENTATION OF VEHICLE TRACKING}

Fig. 7 shows the flow chart for proposed model.

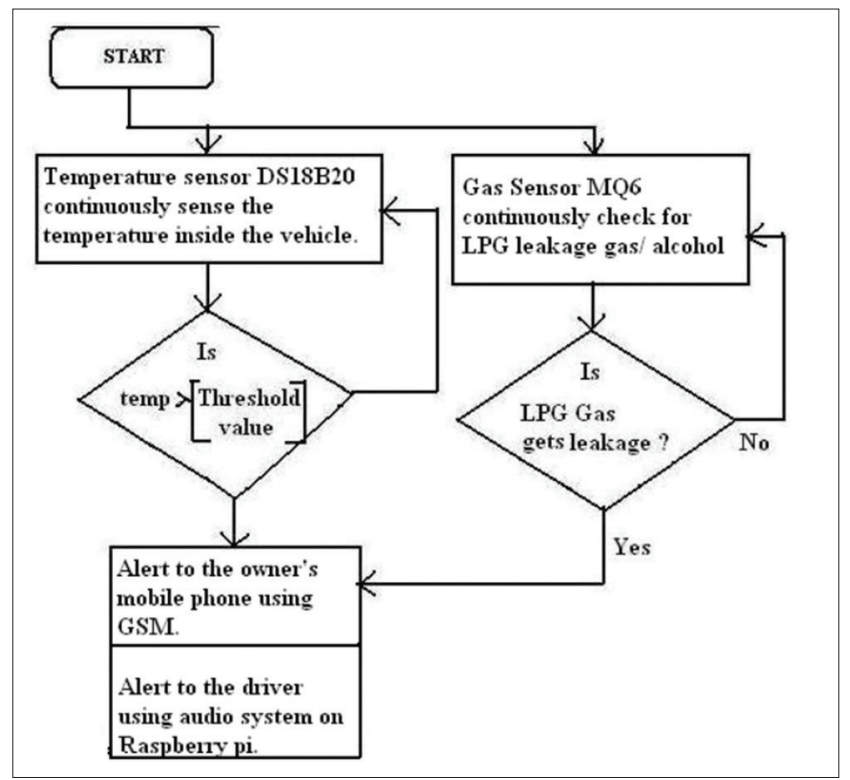

Fig. 7: Flow chart for proposed system

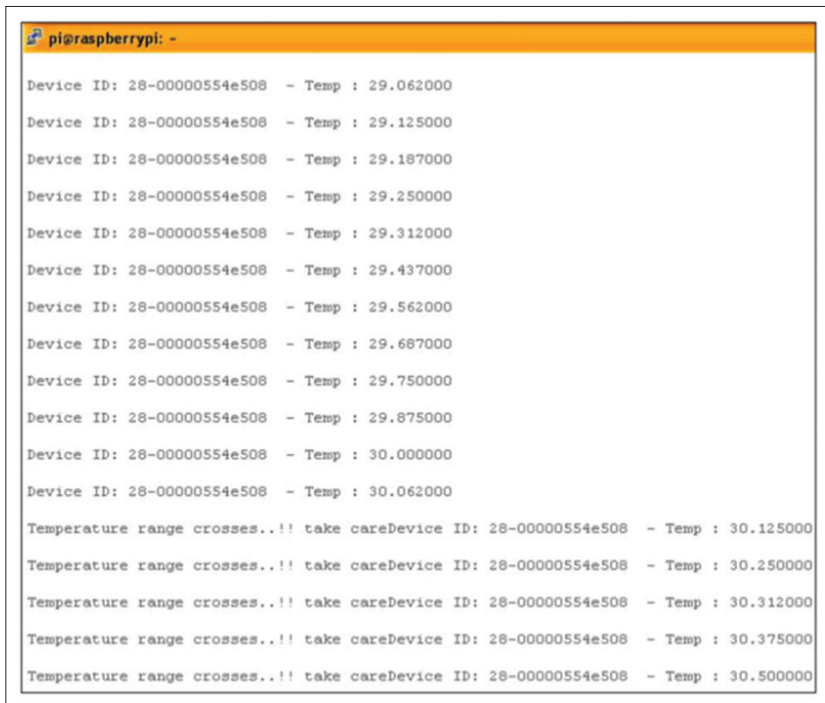

Fig. 8: Results testing for DS18B20 temperature sensor 


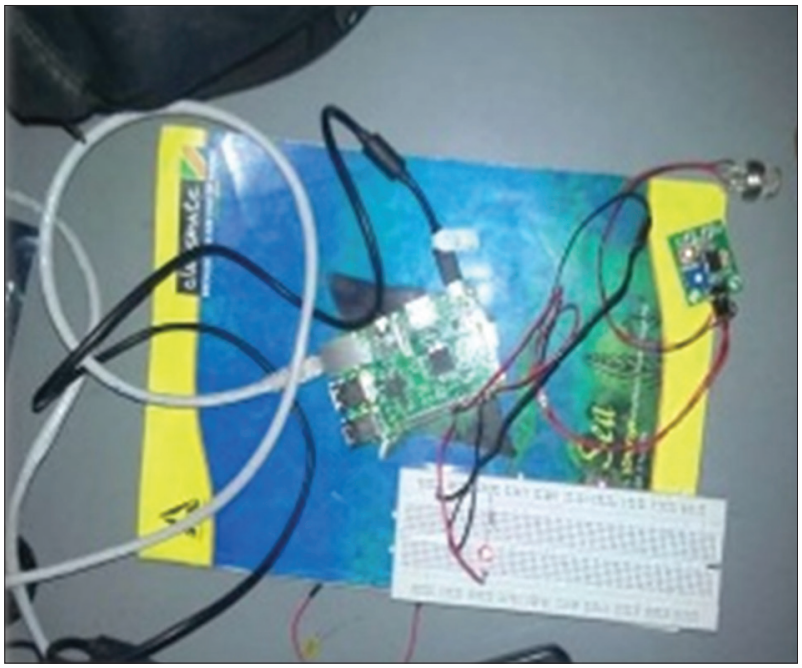

Fig. 9: Results of the MQ 135 gas sensor

\section{TESTING THE RESULTS}

\section{Examining of DS18B20 temperature sensor outputs through the PI board}

This sensor examines the output results which are given in below: The DS18B20 temperature sensor (Fig. 2) depends entirely with the help of 1-wire communication protocol, this sensor provides digital output information when it takes input voltage from the Pi set-up and gets saved in the design entity. Using this setup arrange, the sensor acquires the data easily and fixed further for providing quick warning message. The output of temperature sensor is taken as shown in Fig. 8. Here, while achieving the sensors proving, major controversies to be taken especially:

- Raspberry Pi's (Fig. 4) GPIO is computable with logic level of $3.3 \mathrm{~V}$. Hence, the sensors yield requires to be restrained at acquire logic level

- Things necessary for the prospective setup requires to be selected according to the GPIO pins of the Raspberry Pi's and various combined logic compatibility levels

- The power supply connected to the Raspberry Pi gives voltage and current levels that acquires to be preferred.

\section{Examining the results of gas sensor}

i. The MQ 135 gas sensor gives output (Fig. 9) by taking the input as $5 \mathrm{~V}$

ii. It gives the digital output when it senses the gas

iii. These digital outputs are driven to the Raspberry Pi through the resistor

iv. The GPIO status is to be checked in the Raspberry Pi set-up (Fig. 9), and gas leakage alert message sends to the user mobile through the GSM.

\section{CONCLUSION}

These prospective setups made better usage of tracking machinery by giving alert and secure travelling for the passengers using bad attentive mechanism path. This setup plays a crucial act in real time vehicle capturing of a motion by giving acquired data on the server side at the regular intervals of time to capture the motion regularly. Hence these are the possibilities to trace the motion as quickly as possible in a particular time. Children's alert appliance also takes the provided data through the sensors. In this assured direction, as per the children's concern, the prospective setup also provides alert message to the parent's mobile of accident place and knows about the children daily life activity.

\section{REFERENCES}

1. Kumar H, Kumar R. Availability and Handling of Data Received from the GPS Device: In the Vehicle Tracking. Advance Computing Conference (IACC), IEEE International; 2014. p. 245-9, 21-2.

2. Stalling W. Wireless Communication and Networks. $2^{\text {nd }}$ ed. New Delhi: Prentice Hall of India; 2005

3. Yo O, Polak JW, Noland RW, Park Y, et al. Integration of GPS and dead reckoning for real-time vehicle performance and emission. GPS Solutions. Vol. 4. Berlin: Springer; 1989

4. Tsui JB. Fundamentals of Global Positioning System (GPS) Receivers. $1^{\text {st }}$ ed. Chichester: John Willey \& Sons Inc.; 2000. 